\title{
The Effects of Polydopamine Coated Cu Nanoparticles on the Tribological Properties of Polydopamine/PTFE Coatings
}

\author{
Samuel Beckford ${ }^{\text {a, b) }}$, Leanne Mathurin ${ }^{\text {b, c) }}$, Jingyi Chen ${ }^{\text {b, c) }}$, Robert A. Fleming ${ }^{\text {a, b) }}$, and \\ Min Zou $\left.{ }^{*}, a, b\right)$ \\ a) Department of Mechanical Engineering \\ b) Center for Advanced Surface Engineering \\ University of Arkansas, Fayetteville, AR 72701, USA \\ c) Department of Chemistry and Biochemistry \\ University of Arkansas, Fayetteville, AR 72701, USA
}

* To whom correspondence should be addressed. Tel: (479) 575-6671, Fax: (479) 5756982, E-mail:mzou@uark.edu. 


\begin{abstract}
The effects of using polydopamine (PDA) coated Cu nanoparticles (PDA-Cu) as a filler in the polytetrafluoroethylene (PTFE) topcoat of a PDA/PTFE dual-layer coating is investigated, where the PDA is used as an adhesive basecoat. Tribological tests show that the addition of PDA-Cu in PTFE increases the wear life of PDA/PTFE by a factor of two, approximately three orders of magnitude greater than that of pure PTFE without a PDA basecoat. This increase in wear life is achieved without compromising the low coefficient of friction characteristic of pure PTFE. Scratch tests show that the PDA-Cu filler improves adhesion between the PTFE and the PDA, preventing large scale delamination and also increases the toughness of the coating, preventing ruptures at lower loads.
\end{abstract}

Key words: PTFE; polydopamine; polydopamine coated Cu nanoparticles; wear. 


\section{Introduction}

Polytetrafluoroethylene (PTFE) is a polymer composed of a carbon backbone surrounded by fluorine atoms. It has a high degree of crystallinity $(60-80 \%)$ [1], a melting point of $327^{\circ} \mathrm{C}$, and is commonly used as an engineering material because it is chemically inert, non-toxic, and non-flammable, and exhibits negligible water absorption, anti-stick behavior, high thermal stability, and a low dielectric constant [2]. However, PTFE also has certain disadvantages such as a high wear rate and low load bearing capacity [2], which limit its use in certain applications. In order to overcome these disadvantages, various PTFE compounds have been formed using polymer blends [3, 4], resin binders [5, 6], fibers [7], and nanoparticle fillers [8-10]. Combining these materials with PTFE has proven to increase the crystallinity [11], ultimate strength, elongation to failure, and toughness [12], and as a result, reduces wear in PTFE.

The aim of this study is to examine the tribological effect of incorporating a polydopamine (PDA) adhesive basecoat and PDA coated $\mathrm{Cu}$ nanoparticle filler (PDA$\mathrm{Cu}$ ) in a PTFE coating. PDA-Cu was selected as the filler in this study to take advantage of PDA's adhesion to PTFE. It is expected that the PDA shell in PDA-Cu will first increase the cohesion within the PTFE coating, increasing the toughness of the coated film and as a result preventing rupture of the film at lower loads. Second, it is expected that PDA-Cu will act as discrete nodes of increased adhesion between the PTFE topcoat and the PDA basecoat which will prevent delamination of the PTFE coating at critical loads. Finally, the Cu core in the PDA-Cu filler was selected to further enhance the mechanical properties of the coating. Yan et al. studied Cu nanoparticles in 
PTFE/nano-expanded-graphite and found that $\mathrm{Cu}$ increased the hardness, modulus of elasticity, yield strength, and tensile strength of the composite [13].

Poor adhesion of PTFE coatings to substrates is typically the primary cause of coating failure. Previous studies, carried out by the authors, showed that by adding a PDA adhesive basecoat, the wear life of PTFE coatings was extended 500 times [14]. The adhesive and porous nature of the PDA basecoat allows a thin layer of the PTFE topcoat to sturdily adhere to the substrate while allowing the outermost layers of PTFE to maintain their self-lubricating properties.

The use of PDA as a surface modification method was proposed by Lee et al. [15] as a way to mimic the adhesive proteins secreted by mussels that allows them to stick to both organic and inorganic surfaces, under water, and in tidal conditions [15-17]. PDA is synthesized through $\mathrm{pH}$-dependent oxidation of dopamine hydrochloride in a basic solution, yielding a coating, approximately $50 \mathrm{~nm}$ thick, which can then be coated with various functional coatings. In the field of tribology, coatings such as $\mathrm{ZrO}_{2} / \mathrm{PDA}$ [18], PDA/graphene oxide/PFDTS [19], and PDA/gold on silicon [20] have shown favorable results, producing low friction coatings with extended wear life.

In this study, stainless steel plates coated with a PDA basecoat and a PTFE and PDACu composite topcoat (PDA/PTFE+PDA-Cu) were compared to stainless steel plates coated with a PDA basecoat and a PTFE topcoat (PDA/PTFE). Tribological tests and surface characterization methods were implemented to compare the friction and wear behavior exhibited by the two coating conditions and to identify the wear life, wear mode, surface morphology, and surface chemistry. 


\section{Experimental Details}

The configurations of PDA/PTFE and PDA/PTFE+PDA-Cu samples are depicted in Fig. 1. Three samples were prepared and tested for each condition to produce statistically significant results and ensure repeatability.

\subsection{Materials and Reagents:}

The chemicals used to prepare the PDA-Cu nanoparticles were copper 2,4pentanedionate $\left(\mathrm{Cu}(\mathrm{acac})_{2}, 98 \%\right.$, CAS 13395-16-9, Alfa Aesar), dodecylamine (DDA, 98\%, CAS 124-22-1, Alfa Aesar), butylamine (BTA, 99\%, CAS 109-73-9, Alfa Aesar), dopamine hydrochloride (DA-HCl, 99\%, CAS 62-31-7, Alfa Aesar), tris(hydroxymethyl)aminomethane hydrochloride (Tris-HCl, 99.0\% min, CAS 1185-53-1, $\mathrm{BDH}$ ), and tris(hydroxymethyl)aminomethane (Tris, ultrapure grade, CAS 77-86-1, AMRESCO), and ethanol (200 proof, CAS: $64-17-5$ KOPTEC). All experiments were performed using $18 \mathrm{M} \Omega \mathrm{H}_{2} \mathrm{O}$. All chemicals were used as received.

Cu nanoparticles were synthesized by reduction of $\mathrm{Cu}$ precursors in DDA solution. DDA $(5.5 \mathrm{~g})$ and $\mathrm{Cu}(\mathrm{acac})_{2}(52.5 \mathrm{mg}, 0.2 \mathrm{mmol})$ were added to a $25-\mathrm{mL}$ round-bottom flask equipped with a magnetic stirring bar. The mixture was purged with Ar for 15 min to remove oxygen. The stirring rate was gradually increased to the setting of 5.5 (corning hot stirring plate), while the reaction temperature was increased to $220^{\circ} \mathrm{C}$. The reaction was held at $220{ }^{\circ} \mathrm{C}$ for $25 \mathrm{~min}$ and the $\mathrm{Cu}$ nanoparticles were formed. The reaction solution was then cooled to $180{ }^{\circ} \mathrm{C}$, followed by quenching using ethanol. $\mathrm{Cu}$ nanoparticles were then collected by adding $10 \mathrm{~mL}$ of ethanol to the $5 \mathrm{~mL}$ of reaction solution, followed by centrifuging at 3,300 rcf for $6 \mathrm{~min}$. The $\mathrm{Cu}$ nanoparticles were 
purified twice by a mixture of $2 \mathrm{~mL}$ toluene and $13 \mathrm{~mL}$ of ethanol and recollected by centrifugation at 3,300 rcf for $6 \mathrm{~min}$. The $\mathrm{Cu}$ nanoparticles were then redispersed in 2 $\mathrm{mL}$ toluene for further use.

PDA-Cu nanoparticles were synthesized by adding $0.75 \mathrm{~mL}$ of $\mathrm{Cu}$ nanoparticles ( 1 $\mathrm{mg} / \mathrm{mL}$ ) to a solution containing $1 \mathrm{~mL}$ of water, $1 \mathrm{~mL}$ of ethanol and $0.5 \mathrm{~mL}$ of $1 \mathrm{M}$ Tris$\mathrm{HCl}$ in a $2 \mathrm{dr}$ vial equipped with a magnetic stirring bar. To initiate the coating process, 6 mg of $\mathrm{DA}-\mathrm{HCl}$ was added to the vial. The mixture was stirred at $25{ }^{\circ} \mathrm{C}$ overnight. The reaction mixture turned from light reddish brown to dark brown, indicating the formation of PDA. The PDA coated Cu nanoparticles were collected by centrifuging at 12,000 rcf for $25 \mathrm{~min}$, purified once by ethanol, and redispersed in water. The Cu nanoparticles had an average diameter of $\sim 10 \mathrm{~nm}$ and the PDA coated $\mathrm{Cu}$ nanoparticles had an average diameter of $\sim 120 \mathrm{~nm}$.

The chemicals used to prepare dip coating solutions for fabricating PDA/PTFE coatings were dopamine hydrochloride (H8502, Sigma Aldrich, St. Louis, MO, USA), tris(hydroxymethyl)aminomethane (T1503, Sigma Aldrich, St. Louis, MO, USA), and PTFE 60 wt.\% aqueous dispersion (Teflon Dispersion DISP30, Fuel Cell Earth, MA, USA).

\subsection{Sample Preparation}

The substrate used for coating deposition in this study was type 316 polished stainless steel sheets $305 \times 305 \times 0.76 \mathrm{~mm}(9759 \mathrm{k} 31$, McMaster-CARR, USA). The sheets were first cut into $50 \times 50 \mathrm{~mm}$ squares using a water jet cutter. Subsequently, the squares were soaked in acetone in an ultrasonic bath for 20 minutes to remove oils and organic 
contaminants. Next, the squares were soaked in isopropyl alcohol in an ultrasonic bath for $5 \mathrm{~min}$, and rinsed in deionized water. Finally, the squares were dried using nitrogen gas before beginning the coating deposition process.

The PDA basecoat was deposited by submerging the substrate in an alkaline solution of dopamine hydrochloride for 24 hours [14, 15, 21]. To prepare the dipping solution containing suspended PTFE and PDA-Cu, PDA-Cu nanoparticles were mixed with a PTFE aqueous dispersion to yield a mixture containing 38 wt.\% PTFE and 0.01 wt.\% PDA-Cu. The PDA coated samples were then dipped into either a 38 wt.\% PTFE solution or 38 wt.\% PTFE + 0.01 wt.\% PDA-Cu solution at an insertion/withdrawal speed of $10 \mathrm{~mm} / \mathrm{min}$ using a dip coater (KSV DC, KSV NIMA, USA) to create the PDA/PTFE or PDA/PTFE+PDA-Cu coatings.

Sample preparation was finalized by using a heat treatment process to sinter the coatings to the substrate. The samples were first heated to a temperature of $120^{\circ} \mathrm{C}$ for 3 minutes to remove water from the coating. This step was followed by heating at 300 ${ }^{\circ} \mathrm{C}$ for 4 min to remove wetting agents form the coating, and finally, heating at $372{ }^{\circ} \mathrm{C}$ for 4 min to sinter the PTFE particles to the PDA coated substrate [22, 23].

\subsection{Tribological Testing}

The tribological tests were performed using a linear reciprocating ball-on-flat configuration, with $7 \mathrm{~mm}$ diameter $\mathrm{Cr}$ steel balls, $50 \mathrm{~g}$ load, $2.5 \mathrm{~mm} / \mathrm{s}$ sliding speed, and $15 \mathrm{~mm}$ stroke length on an Automatic Friction Abrasion Analyzer (TS501, Kyowa Interface Science Co., Ltd., Japan). At this load, the Hertzian contact pressure produced on the sample surface was calculated to be $0.45 \mathrm{GPa}$ without considering the coating. 
The average static and dynamic coefficients of friction (COF) were automatically measured for each cycle. A sharp increase in the COF was used as an indicator of coating failure and the number of cycles before the sharp increase was used as a representation of the durability or wear life of the coating.

Tribological tests were also carried out for specific durations (1, 10, 50, 100, 500, 1000 cycles) to observe the wear progression at each of these stages during tribological testing. The wear depth at each of these testing durations was measured to estimate the wear progression over time.

Variable load scratch tests were performed on a Nano + Micro Tribometer (UMT-2, Bruker, Santa Barbara, CA) to identify critical loads at which the coating fails. The scratch tests were carried out by linearly increasing the normal load from 0.1 to $8 \mathrm{~N}$ for a scratch length of $15 \mathrm{~mm}$ and sliding speed of $0.1 \mathrm{~mm} / \mathrm{s}$. Constant load scratches were also performed on each coating at $1-4 \mathrm{~N}$ loads using the same scratch length and sliding speed as the linearly increasing load scratches. The counterface used for all scratches was a $7 \mathrm{~mm}$ diameter $\mathrm{Cr}$ steel ball.

\subsection{Sample Characterization}

To characterize the surface topography, morphology, chemistry, and the effect of tribological tests on these properties of the coating surface, a Surface Stylus Profilometer (Dektak 150, Bruker, Santa Barbara, CA), an Atomic Force Microscope (AFM, Dimension Icon, Bruker, Santa Barbara, CA), a 3D Laser Scanning Confocal Microscope (VK-X100, Keyence Corporation of America, USA) and X-Ray Photoelectron Spectroscopy (PHI 5000 VersaProbe, ULVAC-PHI, Kanagawa, Japan) 
were used. The surface stylus profilometer was used to measure the coating thickness as well as the cross sectional profile of wear tracks produced as a result of the tribological tests. The coating thickness was determined by scanning across the leading edge of the coating and measuring the step height between the bare substrate and the coating. The cross sectional profiles of the wear tracks were measured by scanning across the wear track in a direction perpendicular to the sliding direction of the tribological test. The coating thickness and profiles were measured using a $12.5 \mu \mathrm{m}$ radius stylus with $3 \mathrm{mg}$ contact force, $600 \mu \mathrm{m}$ scan length, and scan duration of 20 seconds.

The AFM and 3D laser microscope were used to characterize the surface topography and morphology at different magnifications. The AFM images provided a depiction of how the surface morphology changed after heat treatment and allowed for a comparison of surface roughness for each sample type. The 3D laser microscope was used to capture images of the scratch tests performed on the samples and identify coating failure modes.

XPS was used to determine the chemical composition of the pristine surface of the coatings, as well as within the wear tracks. Because the coatings are dual layer, XPS scans within the wear tracks were useful to determine whether the basecoat was exposed as a result of coating removal/wear resulting from the tribological tests. Additionally, high resolution C1s scans within the wear tracks were used to examine tribo-induced chemical changes to the material. The XPS spectra were obtained using a monochromatic Al $\mathrm{K}_{\alpha}(1486.6 \mathrm{eV})$ source with a $50 \mu \mathrm{m}$ spot size. Because PTFE is an insulator, dual-beam neutralization was used to avoid charge build up on the samples. 
All spectra were shifted based on a $-\left(\mathrm{CF}_{2}-\mathrm{CF}_{2}\right)$ - peak at a binding energy of $291.0 \mathrm{eV}$ to allow for comparison between spectra.

\section{Results and Discussion}

\subsection{Topographical Characterization}

To examine topographical changes resulting from the heat treatment procedure, AFM images were captured before and after heating the samples to $372{ }^{\circ} \mathrm{C}$. Before heating, AFM images reveal a surface covered with PTFE nanoparticles of 50 to $300 \mathrm{~nm}$ (Fig. 2 (a)). After heating, both PDA/PTFE and PDA/PTFE+PDA-Cu show a similar topography composed of interwoven strands of PTFE (Fig. 2 (b),(c)), indicating that PTFE particles have coalesced to form a more cohesive coating [24]. The PDA/PTFE coating has an average surface roughness, $R_{a}$, of $27.8 \mathrm{~nm}$ and root mean square surface roughness, $R_{q}$, of $34.2 \mathrm{~nm}$. PDA/PTFE+PDA-Cu has an $R_{a}$ of $29.3 \mathrm{~nm}$ and $R_{q}$ of $36.2 \mathrm{~nm}$. There is very little difference in the average surface roughness between both coatings and it is evident that the presence of PDA-Cu nanoparticles does not have an observable effect on the surface topography.

Nanoindentation tests were performed on both coating surfaces to determine their hardness. The maximum indentation depth used was less than $10 \%$ of the coating thicknesses to eliminate substrate effect. The results show that there is an increase in the average hardness for the coating containing the PDA-Cu nanoparticle filler. The average hardness for PDA/PTFE was 52.6 MPa and the average hardness for PDA/PTFE+PDA-Cu was 55.3 MPa. However, the standard deviation in the results is 
too large to infer a statistically significant increase in hardness at this very low concentration of filler.

\subsection{Tribological Testing}

Although the surface topography is similar for both coatings, there is a significant difference in wear life. On average, PDA/PTFE coatings of $1.3 \mu \mathrm{m}$ average thickness withstood 10,080 rubbing cycles during the tribological tests (Fig. 3). PDA/PTFE+PDA$\mathrm{Cu}$ of $1.2 \mu \mathrm{m}$ average thickness, on the other hand, withstood an average of 20,165 rubbing cycles (Fig. 3), a wear life two times greater than PDA/PTFE and three orders of magnitude greater than what is typically observed for pure PTFE under these testing conditions. Furthermore, the incorporation of PDA-Cu in the coating did not produce a statistically significant difference in the dynamic COF. PDA/PTFE+PDA-Cu showed an average dynamic COF of 0.072 compared to a COF of 0.069 observed for PDA/PTFE (Fig. 3). The average static COF for PDA/PTFE+PDA-Cu and PDA/PTFE was 0.136 and 0.141 , respectively, with no statistically significant difference.

Tribological tests carried out for $1,10,50,100,500$, and 1000 cycles to observe wear progression revealed that the wear rate of the topcoat is significantly reduced by incorporating PDA-Cu nanoparticles. The coating thickness for the PDA/PTFE sample used for the wear progression tests was $1.06 \mu \mathrm{m}$. Cross sectional profiles of the wear tracks for each of the testing durations show an incremental progression of wear depth from 1 to 100 cycles, at which point the wear rate significantly decreases and almost comes to a complete standstill after 500 cycles (Fig. 4 (a)). The cross sectional profile of PDA/PTFE at 500 and 1000 cycles revealed a constant wear depth of $0.84 \mu \mathrm{m}$, 
indicating that approximately $0.22 \mu \mathrm{m}$ of coating remained present. As was observed in prior investigations carried out by the authors, a thin layer of PTFE that has strong adhesion to the PDA basecoat remains present for the majority of the testing duration and is responsible for the long wear life of the coating [14].

The PDA/PTFE+PDA-Cu nanoparticle composite coating used in the wear progression tests had a thickness of $1.2 \mu \mathrm{m}$ and showed progressive wear until a wear depth of 0.42 $\mu \mathrm{m}$ was reached after 100 rubbing cycles (Fig. 4 (b)), approximately half that of PDA/PTFE at the same testing stage. At this depth, the wear rate decreases to almost zero (showing very little change between 100, 500 and 1000 cycles) with approximately $0.78 \mu \mathrm{m}$ of coating still remaining. This indicates that at this depth PTFE particles experience a stronger adhesion to the basecoat. The PDA-Cu nanoparticles within the PTFE topcoat act as bolts by allowing discrete regions of stacked PDA layers (Fig. 1) to form at the interface between the basecoat and the topcoat. These stacked regions allow a thicker layer of PTFE topcoat to experience strong adhesion to the substrate. The improved adhesion between the basecoat and topcoat contributes to the increase in wear life observed in PDA/PTFE+PDA-Cu

The wear depth for $1,10,50,100,500$, and 1000 cycles for both coating conditions is plotted in Fig. 5. The plot clearly shows the change in wear rate in both coatings over progressive rubbing cycles. The wear rate for PDA/PTFE has three stages. From 1 to 100 cycles, the wear depth increases at a rate of $77 \mathrm{~nm}$ per test cycle. After 100 cycles, the wear depth increases at a significantly reduced rate of $0.2 \mathrm{~nm}$ per cycle. Finally, after 500 cycles, the wear depth of PDA/PTFE levels off at approximately $0.84 \mu \mathrm{m}$. For PDA/PTFE+PDA-Cu, the wear rate is significantly lower than that of PDA/PTFE. From 1 
to 100 cycles, the wear depth increases at a rate of $41 \mathrm{~nm}$ per test cycle, at which point it levels off at a total wear depth of approximately $0.42 \mu \mathrm{m}$.

\subsection{Chemical Analysis}

A chemical analysis of the surface was carried out to reveal the chemical composition of the coatings as well as changes produced as a result of the tribological testing. XPS, C1s spectra reveal the presence of $-\left(\mathrm{CF}_{3}-\mathrm{CF}_{2}\right)$ - at a binding energy of $292.0 \mathrm{eV}$ and $\left(\mathrm{CF}_{2}-\mathrm{CF}_{2}\right)$ - at a binding energy of $291.0 \mathrm{eV}$ on the pristine surface of PDA/PTFE (Fig. 6 (a)). These peaks are characteristic of PTFE and suggest that the PDA basecoat does not produce any chemical changes to the outermost layers of the PTFE topcoat. Inside the wear track produced in the 1000 cycle tribological test (Fig. 6 (b)), five new peaks are observed at $289.1 \mathrm{eV}, 288.6 \mathrm{eV}, 287.4 \mathrm{eV}, 286.2 \mathrm{eV}$, and $284.8 \mathrm{eV}$. These peaks represent -(CF-CF)-, C=O, C-N, C-O, and adventitious carbon, respectively. -(CF-CF)is produced as a result of scission of the PTFE polymer chain caused by the high pressure tribological test [25]. $\mathrm{C}=\mathrm{O}, \mathrm{C}-\mathrm{N}$, and $\mathrm{C}-\mathrm{O}$ are attributed to PDA [26], revealing that the PDA basecoat may be partially exposed after 1000 cycles. This observation is also supported by the change in color at the center of the wear track shown in the Scanning X-ray Induced secondary electron image inset in Fig. 6 (b).

For PDA/PTFE+PDA-Cu, XPS C1s spectra reveal the presence of $-\left(\mathrm{CF}_{3}-\mathrm{CF}_{2}\right)$ - and $\left(\mathrm{CF}_{2}-\mathrm{CF}_{2}\right)$ - peaks in the pristine surface (Fig. 6 (c)) as was observed for PDA/PTFE. However, the pristine surface of this coating also shows peaks at $288.6 \mathrm{eV}, 287.4 \mathrm{eV}$, $286.2 \mathrm{eV}$, and $284.8 \mathrm{eV}$, which represent $\mathrm{C}=\mathrm{O}, \mathrm{C}-\mathrm{N}, \mathrm{C}-\mathrm{O}$, and adventitious carbon, respectively [26]. These peaks are attributed to PDA and confirm the presence of PDA$\mathrm{Cu}$ in the PTFE topcoat. Inside the 1000 cycle wear track, the same peaks are present 
with the added presence of a peak at $289.1 \mathrm{eV}$, representative of -(CF-CF)- and a result of chain scission as was observed for PDA/PTFE.

Table 1 shows the proportion of $\mathrm{C}, \mathrm{F}, \mathrm{N}$ and $\mathrm{O}$ on both sample surfaces at different rubbing durations. The increasing $\mathrm{N}$ and $\mathrm{O}$ concentration in PDA/PTFE after 100 cycles is evidence that the basecoat is exposed at this stage and remains exposed for the remainder of the tribological test. PDA/PTFE+PDA-Cu, on the other hand, has relatively constant concentrations of $\mathrm{N}$ and $\mathrm{O}$ at different stages of tribological testing. The presence of $\mathrm{N}$ and $\mathrm{O}$ confirms the identification of $\mathrm{C}-\mathrm{N}$ and $\mathrm{C}-\mathrm{O}$ peaks in the $\mathrm{C} 1 \mathrm{~s}$ spectra which are attributed to the PDA-Cu nanoparticles.

\subsection{Scratch Test}

Variable load scratch tests were performed to determine the critical loads impacting the wear mode and wear rate of the coating. The scratch test on PDA/PTFE revealed two critical loads at the applied loading rate and sliding speed. Between 0 and $5.3 \mathrm{~mm}$, PDA/PTFE experiences signs of abrasive wear characterized by removal of surface asperities and compacting of the coating (Fig. 7, (a)). The COF steadily decreases from 0.1 to 0.035 in this running-in period (Fig. 7, (c)). At a load of $3 \mathrm{~N}$ and a distance of 5.3 $\mathrm{mm}$, the PDA/PTFE coating exhibits a large step increase in friction, bringing the COF from 0.035 to 0.055 (Fig. 7, (c)). At this load the coated film ruptures along the edges of the contact area (Fig. 7, (d) a1). At a load of $5.5 \mathrm{~N}$ and a distance of approximately 10.2 $\mathrm{mm}$, the coating suffers large scale delamination characteristic of adhesive failure (Fig. 7, (d) a2). 
PDA/PTFE+PDA-Cu, on the other hand, shows a critical point at a $1.1 \mathrm{~N}$ load and 1.7 $\mathrm{mm}$ distance where there is a sharp peak in the COF that quickly drops down and settles at 0.05 (Fig. 7 (c)). At this point the coated film deforms under the load but shows no sign of rupture (Fig. 7, (d) b1). At a load of $5 \mathrm{~N}$ the COF begins to show larger fluctuations (Fig. 7 (c)) and the coating ruptures along the edges of the contact area (Fig. 7, (d) b2). However, the coating does not suffer large scale delamination for the full load range of the test. The sudden deformation of the PDA/PTFE+PDA-Cu film at a load of $1.1 \mathrm{~N}$ indicates that the presence of the filler may create voids within the film, producing a buckling effect at this load. However, the absence of film rupture below $5 \mathrm{~N}$ and the absence of delamination for the entire test indicate that the PDA-Cu particles, even at only $0.01 \mathrm{wt} . \%$, have a large impact on the film toughness and adhesion to the substrate.

Constant load scratch tests were also performed to confirm the results observed in the variable load scratch tests. Because constant load is applied for the entire $15 \mathrm{~mm}$ scratch, rupture and delamination is observed at slightly lower loads than was seen in the variable scratch test. PDA/PTFE shows signs of film rupture at a load of $3 \mathrm{~N}$ (delineated in red in Fig. 8) and large scale delamination at 4 N. PDA/PTFE+PDA-Cu shows signs of rupture at a load of $4 \mathrm{~N}$ (delineated in red in Fig. 8) and no evidence of delamination. These results confirm that PDA-Cu enhances the toughness of the film as well as increasing the adhesion between the PTFE topcoat and the PDA basecoat.

A small difference in wear track width is also observed between PDA/PTFE and PDA/PTFE+PDA-Cu for each of the constant load scratches (Fig. 8). The difference in width between the two coating conditions points to a change in stiffness of the film. It is 
likely that higher concentrations of the nanoparticle filler will show a more pronounced change in mechanical properties such as these, and, as the synthesis process of the filler is optimized, it is the intent of the authors to evaluate the incorporation of the filler at higher concentrations.

\section{Conclusion}

PDA/PTFE coatings of $1.3 \mu \mathrm{m}$ average coating thickness exhibited an average wear life of 10,082 cycles, approximately 500 times greater than what the authors have observed for pure PTFE coatings. PDA/PTFE+PDA-Cu coatings of $1.2 \mu \mathrm{m}$ average coating thickness exhibit an average wear life of 20,165 cycles, a wear life that is three orders of magnitude greater than pure PTFE. Examination of the wear profile in PDA/PTFE+PDA$\mathrm{Cu}$ coatings at various stages of tribological testing suggests that the presence of the PDA-Cu filler in the PTFE matrix enhances the toughness of the film and increases adhesion to the PDA basecoat.

Future research will focus on optimization of filler concentration. This research direction will allow a better understanding of the role the nanoparticles play in the durability and friction exhibited by the coating.

\section{Acknowledgments}

We thank the Arkansas Biosciences Institute and the University of Arkansas for major equipment funding support. We also thank the Arkansas Nano \& Bio Materials Characterization Facility for equipment use and the University of Arkansas for funding support. We acknowledge partial support from the Center for Advanced Surface 
Engineering, under the National Science Foundation Grant No. OIA-1457888 and the Arkansas EPSCoR Program, ASSET III.

\section{References}

[1] Song F, Wang Q, Wang T. The effects of crystallinity on the mechanical properties and the limiting PV (pressure xvelocity) value of PTFE. Tribol. Int. 2016; 93, Part A:1-10.

[2] Venkateswarlu G, Sharada R, Rao MB. Polytetrafluoroethylene (PTFE) based composites. J. Chem. Pharm. Res. 2014; 6:508-17.

[3] Hu F, Wen X, Pi P, Cheng J, Yang Z. Preparation of copper clad laminates based on polytetrafluoroethylene with blending modification. Huanan Ligong Daxue Xuebao/Journal of South China University of Technology (Natural Science) 2005; 33:31-6.

[4] Burris DL, Sawyer WG. Tribological behavior of PEEK components with compositionally graded PEEK/PTFE surfaces. Wear 2007; 262:220-4.

[5] Carton J-, Vannes A-, Zambelli G, Vincent L. Investigation of the fretting behaviour of low friction coatings on steel. Tribol. Int. 1996; 29:445-55.

[6] McCook NL, Burris DL, Bourne GR, Steffens J, Hanrahan JR, Sawyer WG. Wear resistant solid lubricant coating made from PTFE and epoxy. Tribol. Lett. 2005; 18:11924.

[7] Qian-qian S, Xian-hua C. On the friction and wear behavior of PTFE composite filled with rare earths treated carbon fibers under oil-lubricated condition. Wear 2006; 260:1243-7.

[8] Kandanur SS, Schrameyer MA, Jung KF, Makowiec ME, Bhargava S, Blanchet TA. Effect of activated carbon and various other nanoparticle fillers on PTFE wear. Tribol. Trans. 2014; 57:821-30.

[9] Sawyer WG, Freudenberg KD, Bhimaraj P, Schadler LS. A study on the friction and wear behavior of PTFE filled with alumina nanoparticles. Wear 2003; 254:573-80.

[10] Burris DL, Sawyer WG. Improved wear resistance in alumina-PTFE nanocomposites with irregular shaped nanoparticles. Wear 2006; 260:915-8.

[11] Burris DL, Zhao S, Duncan R, Lowitz J, Perry SS, Schadler LS et al. A route to wear resistant PTFE via trace loadings of functionalized nanofillers. Wear 2009; 267:653-60. 
[12] Burris DL, Effects of nanoparticles on the wear resistance of polytetrafluoroethylene (Doctoral Dissertation). University of Florida 2007.

[13] Yan Y, Jia Z, Yang Y. Preparation and mechanical properties of PTFE/nano-EG composites reinforced with nanoparticles. Procedia Environ. Sci. 2011; 10:929-35.

[14] Beckford S, Zou M. Wear resistant PTFE thin film enabled by a polydopamine adhesive layer. Appl. Surf. Sci. 2014; 292:350-6.

[15] Lee H. Bioadhesion of mussels and geckos : Molecular mechanics, surface chemistry, and nanoadhesives (Doctoral Dissertation). Northwestern University. Proquest Dissertations Publishing 2008.

[16] Crisp DJ, Walker G, Young GA, Yule AB. Adhesion and substrate choice in mussels and barnacles. J. Colloid. Interface Sci. 1981; 104:40-50.

[17] Waite JH. Adhesion à la moule. Integrative and Comparative Biology 2002; 42:1172-80.

[18] Ou J, Wang J, Qiu Y, Liu L, Yang S. Mechanical property and corrosion resistance of zirconia/polydopamine nanocomposite multilayer films fabricated via a novel nonelectrostatic layer-by-layer assembly technique. Surf. Interface. Anal. 2011; 43:803-8.

[19] Ou J, Liu L, Wang J, Wang F, Xue M, Li W. Fabrication and tribological investigation of a novel hydrophobic polydopamine/graphene oxide multilayer film. Tribol. Lett. 2012; 48:407-15.

[20] Songfeng E, Shi L, Guo Z. Tribological properties of self-assembled gold nanoparticles on silicon with polydopamine as the adhesion layer. Appl. Surf. Sci. 2014; 292:750-5.

[21] Beckford S, Mathurin L, Chen J, Zou M. The influence of Cu nanoparticles on the tribological properties of polydopamine/PTFE + Cu films. Tribol. Lett. 2015; 59:11.

[22] DuPont Fluoroproducts. Teflon PTFE TE3859 Product information. http://www2.dupont.com/Teflon_Industrial/en_US/assets/downloads/k10918.pdf 2006; 2014:4.

[23] Dupont. Dupont Fluoroproducts - Disperssion Properties and Processing Guide. Dupont technical data 2001;RWJ86:1-12.

[24] Beckford S, Cai J, Chen J, Zou M. Use of Au Nanoparticle-Filled PTFE Films to Produce Low-Friction and Low-Wear Surface Coatings. Tribol. Lett. 2014; 56:223-30.

[25] Beckford S, Zou M. Wear resistant PTFE thin film enabled by a polydopamine adhesive layer. Appl. Surf. Sci. 2014; 292:350-6. 
[26] Qiang W, Fulong Z, Jie L, Beijia L, Changsheng Z. Oxidant-induced dopamine polymerization for multifunctional coatings. Polymer Chemistry 2010;1:1430-3.

Table 1 XPS results for chemical element proportions in PDA/PTFE and PDA/PTFE+PDA-Cu after 0, 50, 100, 500, and 1000 rubbing cycles.

\begin{tabular}{|c|c|c|c|c|}
\hline \multicolumn{5}{|c|}{ PDA/PTFE } \\
\hline \multirow{2}{*}{ Surface Condition } & \multicolumn{5}{|c|}{ Chemical Element } \\
\cline { 2 - 5 } & C1s & F1s & N1s & O1s \\
\hline pristine surface & 28.86 & 71.14 & 0.00 & 0.00 \\
\hline 50 cycles & 30.78 & 69.04 & 0.00 & 0.18 \\
\hline 100 cycles & 30.31 & 68.58 & 0.74 & 0.37 \\
\hline 500 cycles & 32.70 & 63.68 & 0.90 & 2.72 \\
\hline 1000 cycles & 33.37 & 63.35 & 1.10 & 2.18 \\
\hline \multicolumn{5}{|c|}{ PDA/PTFE + PDA-Cu } \\
\hline \multirow{2}{*}{ Surface Condition } & C1s & F1s & N1s & $01 s$ \\
\hline pristine surface & 32.39 & 66.85 & 0.56 & 0.20 \\
\hline 50 cycles & 32.49 & 66.91 & 0.43 & 0.17 \\
\hline 100 cycles & 32.35 & 67.10 & 0.35 & 0.20 \\
\hline 500 cycles & 31.70 & 67.45 & 0.40 & 0.45 \\
\hline 1000 cycles & 31.05 & 67.95 & 0.59 & 0.41 \\
\hline
\end{tabular}




\section{List of Figures}

Fig. 1 Schematic of control group, PDA/PTFE and experimental group, PDA/PTFE+PDA-Cu.

Fig. 2 AFM topographical images of (a) PDA/PTFE nanoparticle film, (b) PDA/PTFE nanoparticle film heated to $372^{\circ} \mathrm{C}$ and (c) PDA/PTFE+PDA-Cu nanoparticle composite film heated to $372{ }^{\circ} \mathrm{C}$.

Fig. 3 Wear life and coefficient of friction results for PDA/PTFE and PDA/PTFE+PDA$\mathrm{Cu}$. The bar chart shows averages with individual measurement results shown as asterisks and circles. The average coefficient of friction is calculated from friction values before coating failure.

Fig. 4 Cross-sectional profiles of (a) PDA/PTFE and (b) PDA/PTFE+PDA-Cu after 1, 10, $50,100,500$, and 1000 rubbing cycles.

Fig. 5 Plot of wear depth as a function of rubbing cycles for PDA/PTFE and PDA/PTFE+PDA-Cu after 1, 10, 50, 100, 500, and 1000 rubbing cycles.

Fig. 6 XPS C1s spectra for PDA/PTFE (a) pristine surface and (b) after 1000 rubbing cycles, and for PDA/PTFE+PDA-Cu (c) pristine surface and (d) after 1000 rubbing cycles.

Fig. 7 Optical image of linearly increasing load scratch on (a) PDA/PTFE and (b) PDA/PTFE+PDA-Cu, (c) coefficient of friction and normal load as a function of scratch distance, and (d) optical images of point a1, a2, b2, and b2 as labeled in (a) and (b).

Fig. 8 Constant load scratch test on PDA/PTFE performed under (a) $1 \mathrm{~N}$, (b) $2 \mathrm{~N}$, (c) 3 $\mathrm{N}$, and (d) $4 \mathrm{~N}$ normal loads, and on PDA/PTFE+PDA-Cu performed under (e) $1 \mathrm{~N}$, (f) 2 $\mathrm{N}$, (g) $3 \mathrm{~N}$, and (h) $4 \mathrm{~N}$ normal loads. 


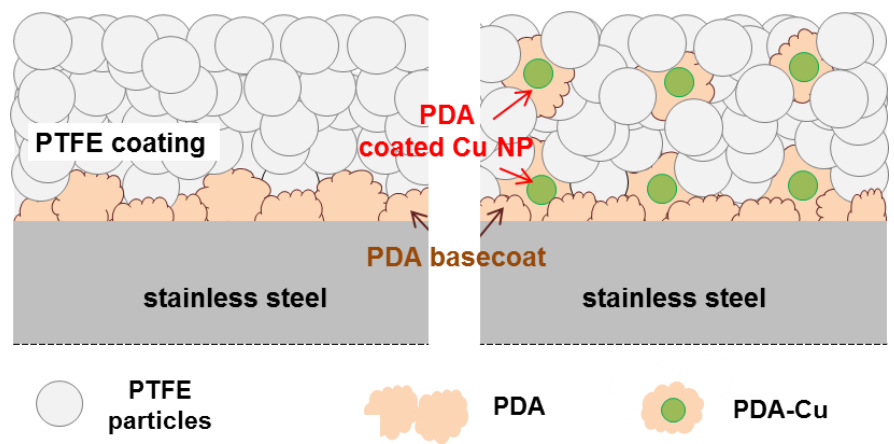

Fig. 1 Schematic of control group, PDA/PTFE and experimental group, PDA/PTFE+PDA-Cu. 

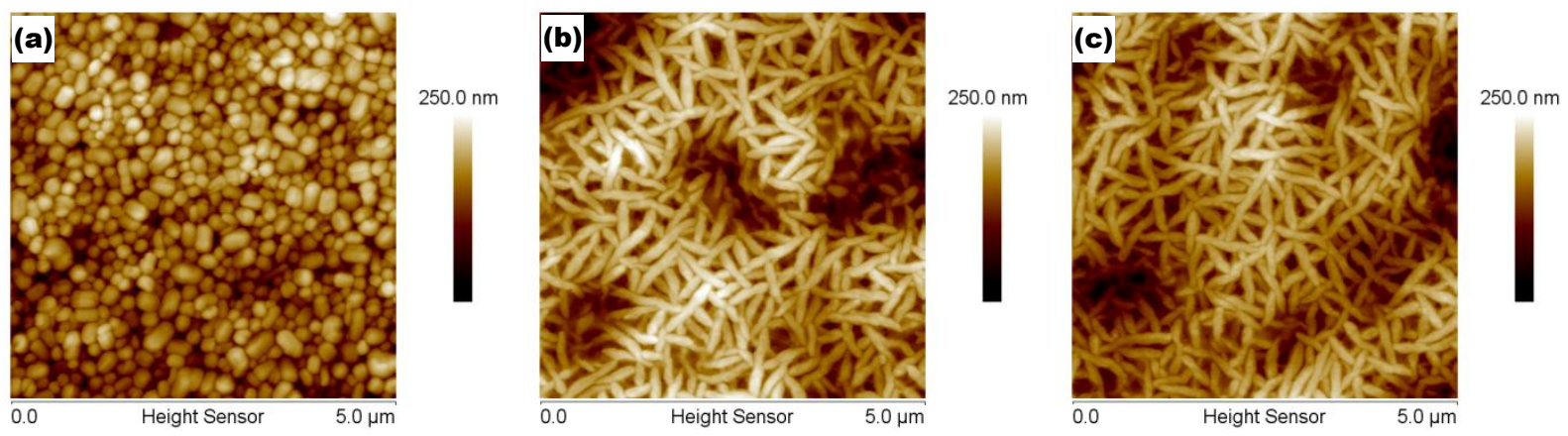

Fig. 2 AFM topographical images of (a) PDA/PTFE nanoparticle film, (b) PDA/PTFE nanoparticle film heated to $372{ }^{\circ} \mathrm{C}$ and (c) PDA/PTFE+PDA-Cu nanoparticle composite film heated to $372{ }^{\circ} \mathrm{C}$. 


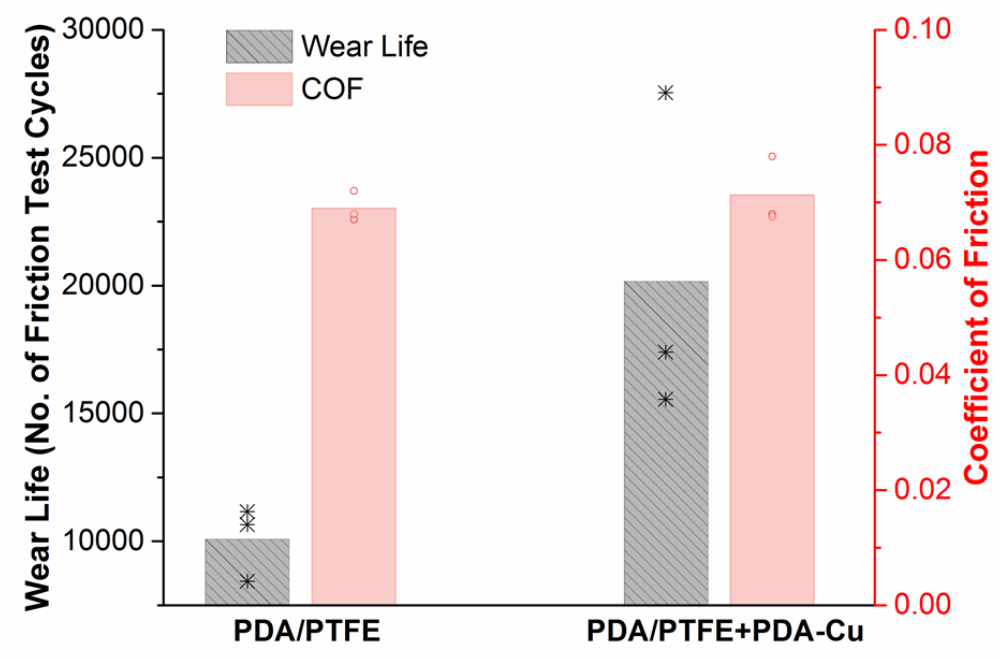

Fig. 3 Wear life and coefficient of friction results for PDA/PTFE and PDA/PTFE+PDA$\mathrm{Cu}$. The bar chart shows averages with individual measurement results shown as asterisks and circles. The average coefficient of friction is calculated from friction values before coating failure. 

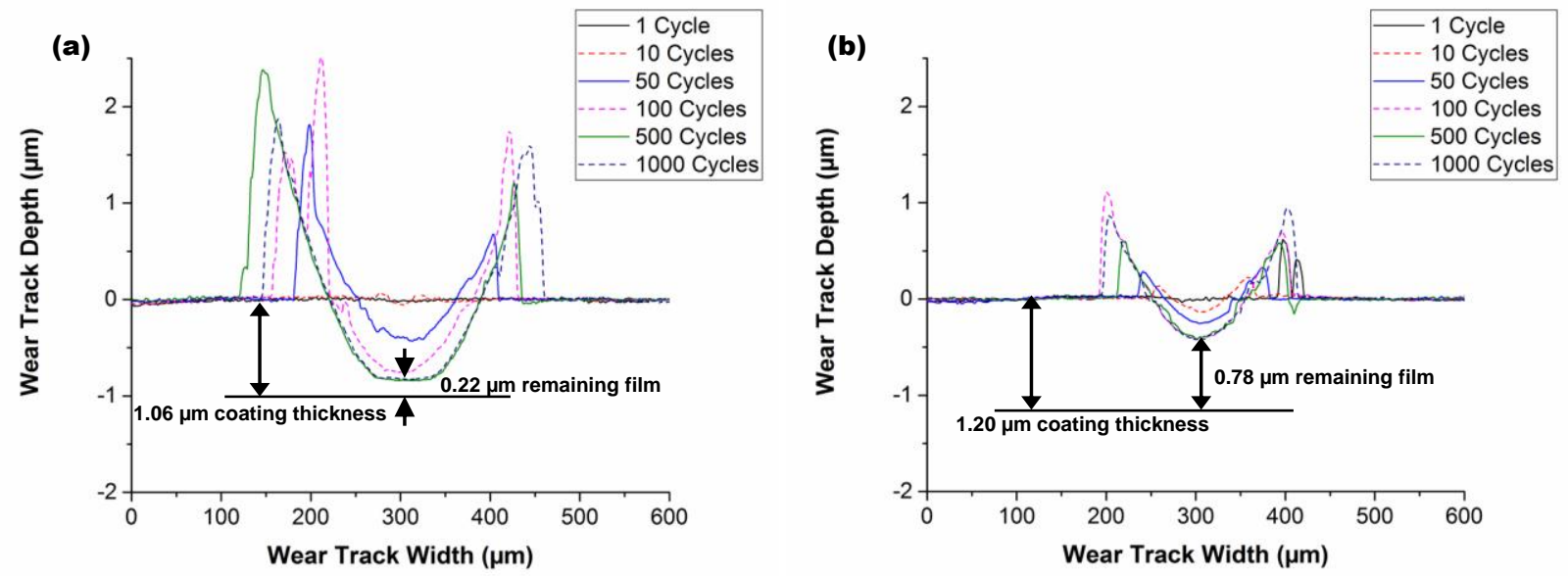

Fig. 4 Cross-sectional profiles of (a) PDA/PTFE and (b) PDA/PTFE+PDA-Cu after 1, 10, 50, 100, 500, and 1000 rubbing cycles. 


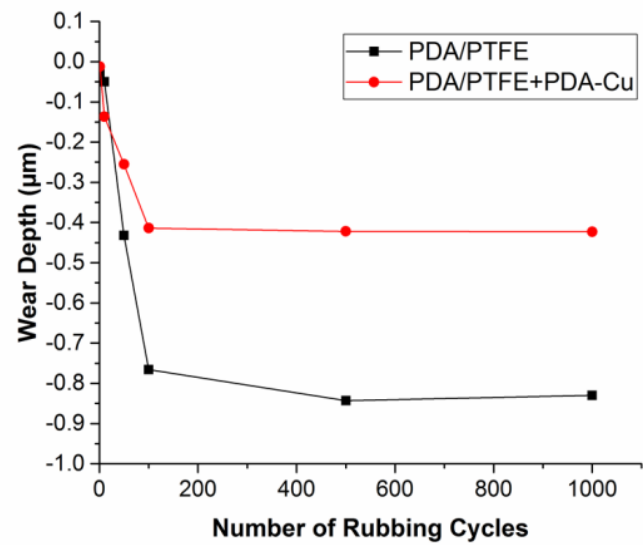

Fig. 5 Plot of wear depth as a function of rubbing cycles for PDA/PTFE and PDA/PTFE+PDA-Cu after 1, 10, 50, 100, 500, and 1000 rubbing cycles. 
(a)
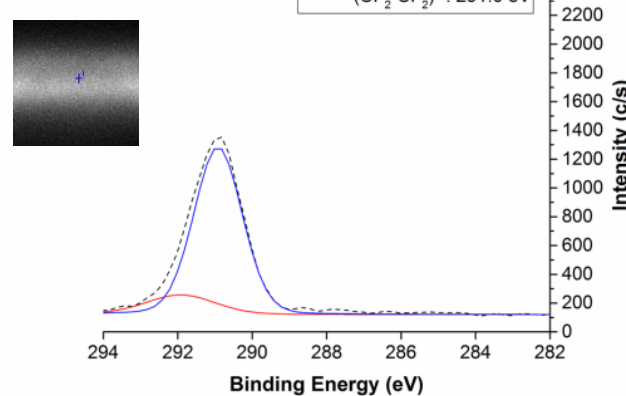

(c)

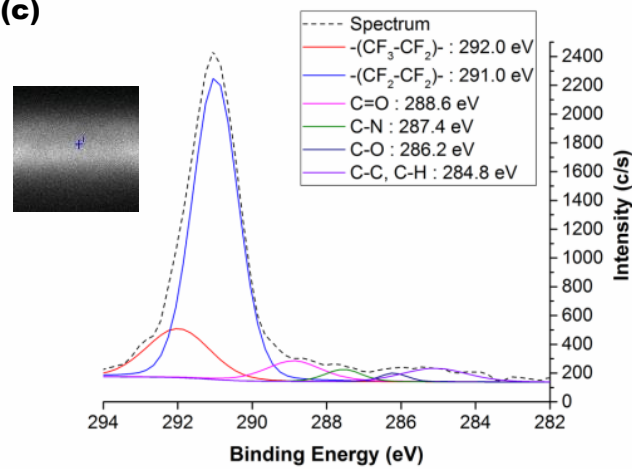

(b)

b)

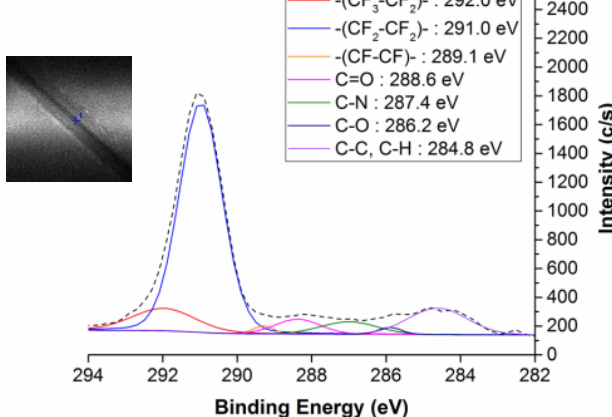

(d)

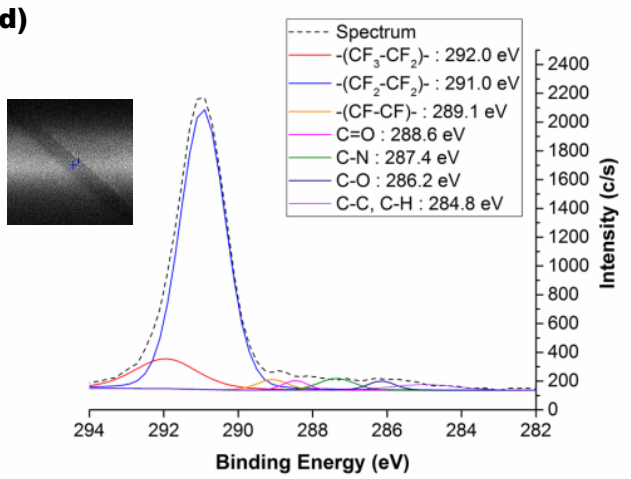

Fig. 6 XPS C1s spectra for PDA/PTFE (a) pristine surface and (b) after 1000 rubbing cycles, and for PDA/PTFE+PDA-Cu (c) pristine surface and (d) after 1000 rubbing cycles. 
(a)

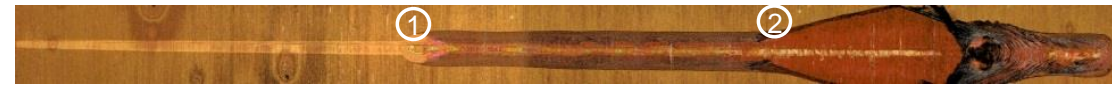

(b)
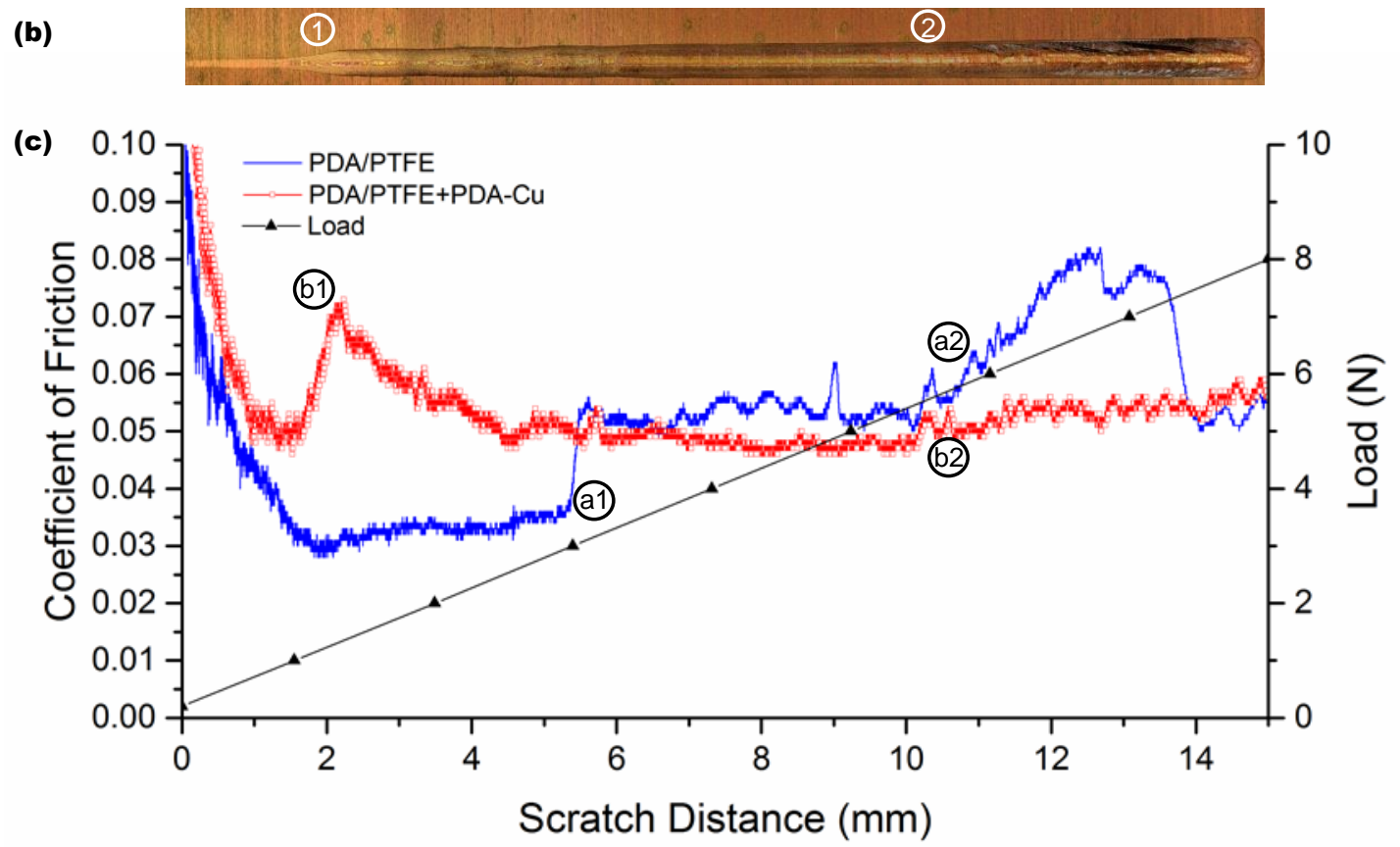

(d)

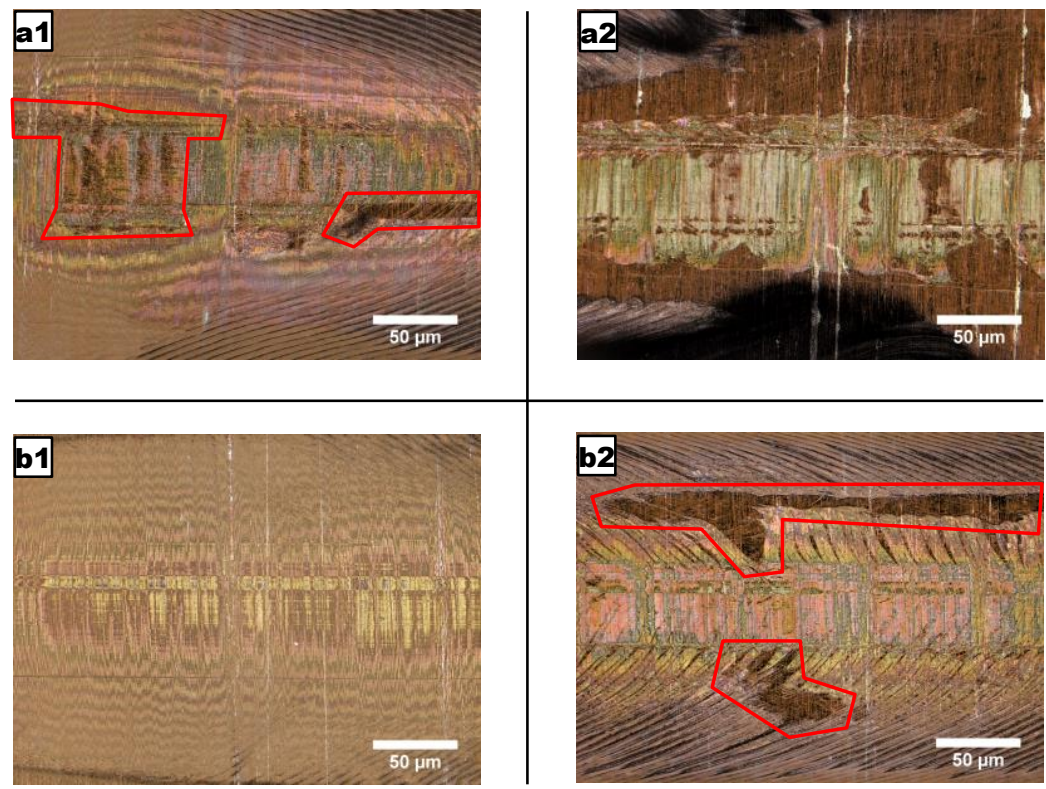

Fig. 7 Optical image of linearly increasing load scratch on (a) PDA/PTFE and (b) PDA/PTFE+PDA-Cu, (c) coefficient of friction and normal load as a function of scratch distance, and (d) optical images of point a1, a2, b2, and b2 as labeled in (a) and (b). 


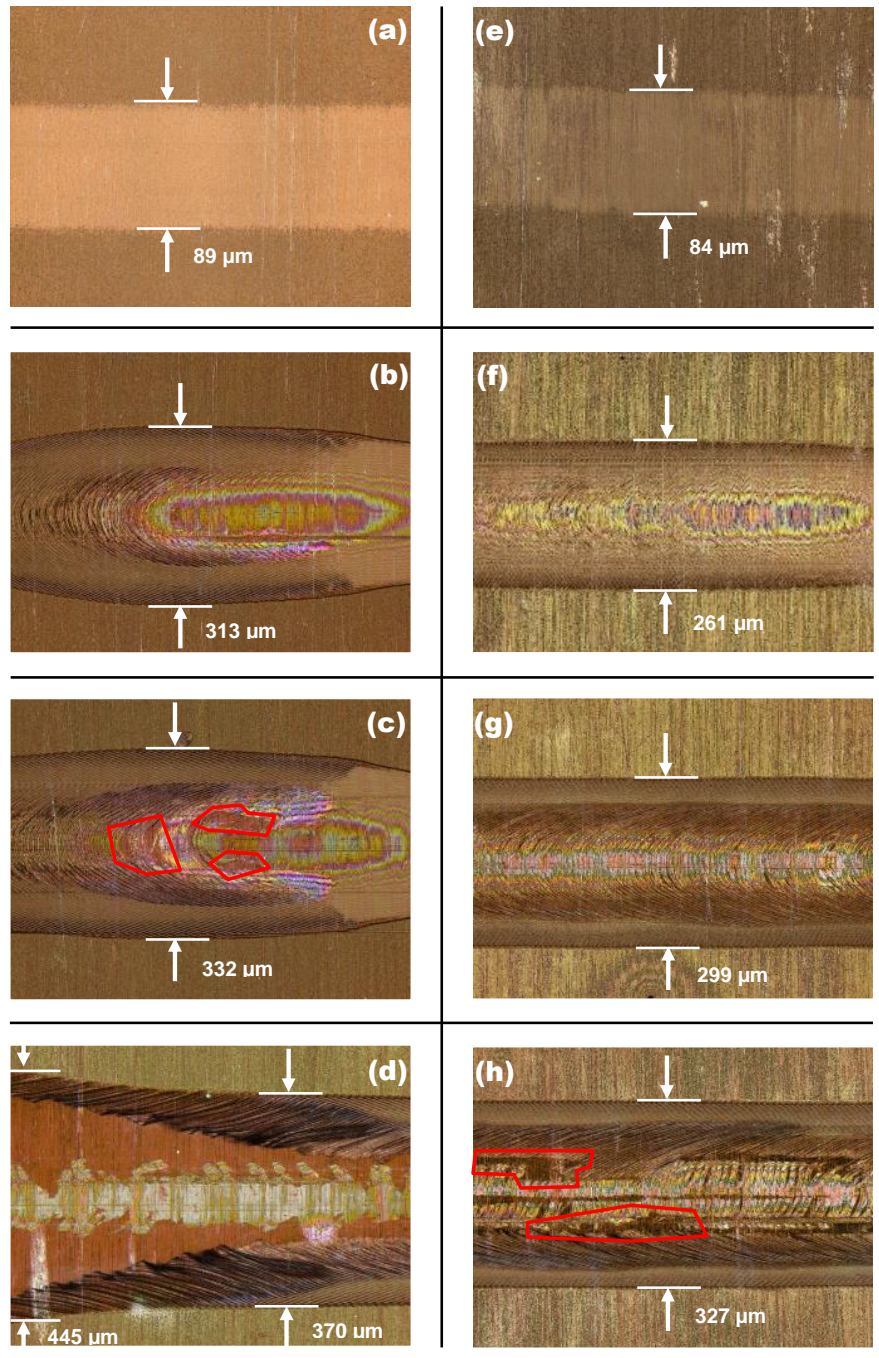

Fig. 8 Constant load scratch test on PDA/PTFE performed under (a) $1 \mathrm{~N}$, (b) $2 \mathrm{~N}$, (c) $3 \mathrm{~N}$, and (d) $4 \mathrm{~N}$ normal loads, and on PDA/PTFE+PDA-Cu performed under (e) $1 \mathrm{~N}$, (f) $2 \mathrm{~N}$, (g) $3 \mathrm{~N}$, and (h) $4 \mathrm{~N}$ normal loads. 